\title{
Amputation neuroma of the middle ear mimicking glomus tympanicum tumor
}

\author{
Meike Schikschneit, MD, Wolfgang Maier, MD, Gian Kayser, MD, \\ Ansgar Berlis, MD, and Carsten Christof Boedeker, MD, Freiburg, Germany
}

O bjective tinnitus is rare compared with the more frequent subjective tinnitus. Objective tinnitus may be due to muscular and vascular pathologies. Muscular tinnitus is usually described as an "ear click" that is caused by myocloni of the stapedial muscle, tensor tympani muscle, or muscles of the epipharynx and soft palate. ${ }^{1}$

Vascular tinnitus, which is generally pulsatile, is usually due to alterations in cervical or cranial blood flow, vascular abnormalities, as well as tumors of the lateral skull base and the middle ear. ${ }^{2}$ Tumors that are frequently characterized by a pulsatile tinnitus (PT) are glomus jugulare (GJ) and glomus tympanicum (GT) tumors. ${ }^{3}$

Amputation neuromas (ANs) are benign tumors secondary to the disordered proliferation of nerves and connective tissue after total or partial dissection of a nerve. ${ }^{4}$ The formation of an AN is an uncommon occurrence after procedures in the head and neck. To the best of our knowledge, an AN in the middle ear has not been described in international medical literature yet.

We present an amputation neuroma of the middle ear mimicking the clinical, audiological, and radiographic features of a glomus tympanicum tumor as a unique cause of a PT.

A 65-year-old male patient presented to our outpatient department in 1999 with a 3-month history of a pulsatile right-sided tinnitus. He had undergone a type I tympanoplasty on the right at another institution in 1992. The otological examination revealed a reddish-blue mass behind an intact right eardrum with pulse synchronous movements. Pure tone audiometry demonstrated a conductive right-sided hearing loss of 5 to $15 \mathrm{~dB}$. MRI and CT showed a mass of the right middle ear that had left the cortical bone over the jugular bulb intact. Digital substraction angiography (DSA) demonstrated a highly vascularized tumor (Fig 1), with the ascending pharyngeal artery being the main feeding vessel. Regarding the examinations mentioned, the preoperative diagnosis was that of a GT tumor type B according to the Fisch classification. The tumor underwent superselective embolization prior to complete resection via a mastoidectomy with posterior tympanotomy.

On histopathological examination, the tumor consisted of spindle-shaped cells. The tumor cells showed no significant

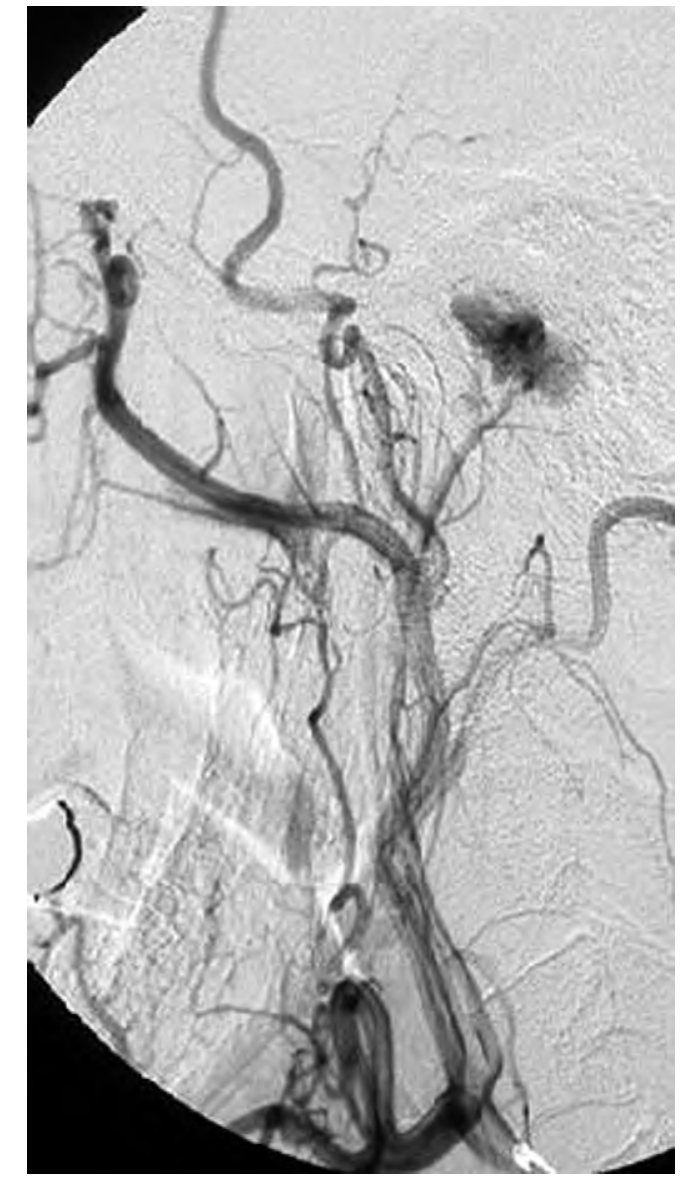

Figure 1 DSA revealing a highly vascularized tumor on the right promontory.

cytological atypia and no mitotic figures. The so-called zellballen pattern that is typical for paragangliomas was not detected. The immunohistochemical profile was negative for chromogranin (Fig 2) and neurone-specific enolase (NSE). The tumor showed a strong expression of the S100 protein though. The histopathological and immunohistochemical profiles corroborated the diagnosis of an amputation neuroma of the middle ear. The subsequent clinical course was uneventful. The PT and the conductive hearing 


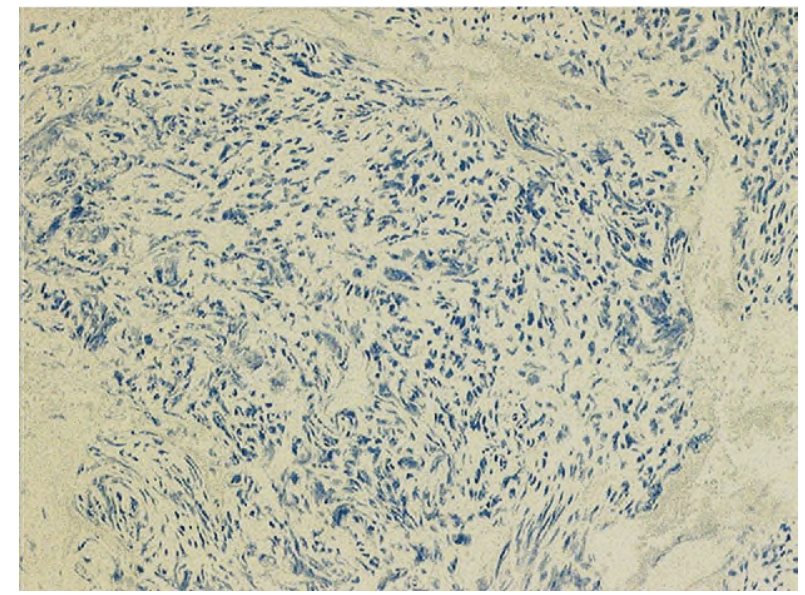

Figure 2 Negative immunohistochemical profile for chromogranin. (Original magnification: $\times 10$.)

loss subsided postoperatively. Unfortunately, the patient has been lost to follow-up in the meantime.

Review of the report of the tympanoplasty in 1992 revealed a strong adhesion of parts of the right eardrum to the promontory. Therefore the tympanoplasty performed in 1992 has to be considered the trigger for the development of the amputation neuroma described.

\section{DISCUSSION}

Tinnitus is a common auditory phenomenon. In the vast majority of cases, it is subjective; that is, it may be noticed by only the patient. Objective tinnitus on the other hand is audible to both the patient and the examiner. ${ }^{1}$

Muscular objective tinnitus may be caused by repetitive contractions of the stapedial muscle or the tensor tympani muscle (middle ear myoclonus). ${ }^{1}$ Another possible cause is a myoclonus of the epipharynx or the soft palate, especially the levator veli palatini muscle characterized by typical ear clicks (palatoclonus). ${ }^{1}$

The etiology of PT has been reviewed by Sismanis. ${ }^{2}$ PT usually originates from vascular structures within the cranial cavity, the head and neck region, or the thoracic cavity, by either increased blood flow or lumen stenosis.

The most common tumors of the lateral skull base and middle ear that typically become symptomatic by means of a PT are GJ and GT tumors. ${ }^{3}$ Both are usually diagnosed on the basis of typical clinical and radiographic findings before treatment is initiated. The tumor presented revealed the standard features of a paraganglioma of the middle ear, that is, typical otological examination, PT with conductive hearing loss, and characteristic radiographic examinations with a highly vascularized tumor on the promontory. Histopathologically, paragangliomas are well-vascularized tumors that consist of two major cell types: chief cells and sustentacular cells. The arrangement of the chief cells in a so-called zellballen pattern is typical for paragangliomas. On immu- nohistochemistry, chief cells are positive for NSE and chromogranin, whereas sustentacular cells show expression of the S100 antigen. ${ }^{5}$ The tumor in our case did not feature chief cells, sustentacular cells, or a zellballen pattern. In addition, there was no expression of NSE or chromogranin.

The formation of amputation neuromas is caused by a proliferation of Schwann cells, axons, and connective tissue elements. ${ }^{5}$ They occur after accidental or surgical trauma in an attempt to reinnervate the area. Although ANs in the head and neck most commonly derive from the greater auricular nerve, they may form at any site and affect any type of nerve. In our patient it is likely that injury of the tympanic plexus on the promontory during the tympanoplasty in 1992 represented the trigger for the formation of the AN.

The case presented is noteworthy for the very unusual site and presentation of an amputation neuroma. Additionally, it represents a very uncommon differential diagnosis of a glomus tympanicum tumor.

\section{AUTHOR INFORMATION}

From the Departments of Otorhinolaryngology-Head and Neck Surgery (Drs Schikschneit, Maier, and Boedeker), Pathology (Dr Kayser), Neuroradiology (Dr Berlis), University of Freiburg.

Corresponding author: Dr Carsten Christof Boedeker, MD, Department of Otorhinolaryngology-Head and Neck Surgery, University of Freiburg, Killianstrasse 5, D-79106 Freiburg, Germany.

E-mail address: boedeker@hno.ukl.uni-freiburg.de.

\section{AUTHOR CONTRIBUTIONS}

Meike Schikschneit, writing of the manuscript; Wolfgang Maier, operation of the patient, thorough reading of the manuscript; Gian Kayser, histopathologic and immunohistochemical studies, thorough reading of the manuscript; Ansgar Berlis, neuroradiological images and embolisation, thorough reading of the manuscript; Carsten Christof Boedeker, writing of the manuscript, idea.

\section{FINANCIAL DISCLOSURE}

None.

\section{REFERENCES}

1. Golz A, Fradis M, Martzu D, et al. Stapedius muscle myoclonus. Ann Otol Rhinol Laryngol 2003;112:522-4.

2. Sismanis A. Pulsatile tinnitus. Otolaryngol Clin N Am 2003;36:389402.

3. Boedeker CC, Ridder GJ, Schipper J. Paragangliomas of the head and neck: diagnosis and treatment. Fam Cancer 2005;4:55-9.

4. Talmi YP, Bedrin L, Dori S, et al. Amputation neuromas after neck dissection. Otolaryngol Head Neck Surg 2003;128:196-9.

5. Shibahara J, Goto A, Niki T, et al. Primary pulmonary paraganglioma: report of a functioning case with immunohistochemical and ultrastructural study. Am J Surg Pathol 2004;28:825-9. 